\title{
El rol docente de una sociedad científica
}

\author{
The academic role of a scientific society
}

\author{
Alejandro Campos Gutiérrez ${ }^{1}$
}

Hace dos años, asumí el Comité de Educación de la Sociedad de Cirujanos de Chile.

Los lineamientos a desarrollar han consistido, en primer lugar, a establecer el nexo con los Residentes de Cirugía del país, a través de su presidente, que en estos años ha sido el Dr. Guillermo Martínez, canalizando sus inquietudes y necesidades, apoyándolos en las distintas actividades en que lo requieran, entre otras, la Seccional de Residentes que se realiza en el Congreso de Cirugía anual; el último evento correspondió al año 2018 y tuvo más de 300 asistentes presenciales.

En segundo lugar, está el promover y difundir la enseñanza de la cirugía, en las distintas instancias académicas del país, creando, promoviendo y apoyando la realización de cursos de cirugía; todo esto, ha sido la tarea encomendada, en forma entusiasta, por el Presidente actual de la Sociedad, Dr. Carlos García y su directorio.

Es así, como hemos desarrollado cursos durante la semana, en días distintos, para diferentes estamentos académicos, con el apoyo tecnológico de la Sociedad de Cirujanos y del Dr. Gabriel Sandoval (Residente de Cirugía, de tercer año, del Hospital Clínico de la Universidad de Chile).

Los días lunes, junto a Dr. Gabriel Sandoval, Dr. Guillermo Martínez, Dr. Marcel Sanhueza e Internos (Marcelo Rojas, Jorge Segovia, Catalina Díaz, y alumnos (Alejandro Campos Sáenz, Javier Toro, Alessandra Jarufe) de Medicina de distintas Universidades del país, creamos el curso "Cirugía para Estudiantes de la Salud ", el cual está orientado para estudiantes de pregrado, de distintas carreras de la salud, de acceso gratuito, previa inscripción, basado en una modalidad interactiva, con preguntas a los asistentes durante el desarrollo de la presentación. La exposición de los temas, está a cargo de reconocidos cirujanos y cirujanas del país; comenzamos en mayo de 2020, en un formato semanal, planificadas
30 sesiones, con término a fines de diciembre del mismo año; este curso logró una inscripción de 3.300 personas, con una asistencia promedio entre 1.500 a 2.500 por sesión, y cuenta como asesor Docente, al Prof. Dr. Attila Csendes, Maestro de la Cirugía Chilena.

Para los días martes, El Dr. Guillermo Martínez, creó el "Curso de Educación Continua, para Residentes de Cirugía General", hemos apoyado esta tremenda iniciativa; este curso, implica presentaciones en distintos tópicos de la Cirugía, con una presentación por parte de un experto (a) en el tema, con 4-6 panelistas, que participan, discuten y resuelven dudas al término de la presentación; generalmente, la asistencia fluctúa entre 450 a 650 personas, en su mayoría, Residentes de Cirugía del país.

El día miércoles, junto al Dr. Sandoval, desarrollamos un curso destinado a los Cirujanos del país, con participación activa de los capítulos regionales, de manera tal que el expositor o expositora, sea generalmente, un miembro de cada capítulo regional, con un panel de expertos (4-6) al término de la presentación, para discutir el tema; este curso tiene una asistencia entre 200-500 personas por sesión.

Esta ardua labor docente de la Sociedad de Cirujanos de Chile, ha sido llevada a cabo, de manera online, con participación en su realización de muchos cirujanos y cirujanas del país, de Arica a Magallanes, además de Residentes y Alumnos (as) de Medicina, en forma desinteresada, lo cual ha permitido el acceso de la enseñanza de la Cirugía, a todos los estudiantes de pregrado y postgrado de nuestro país, y de varios países de Latinoamérica, que han asistido y asisten a nuestros cursos.

Debo agradecer al Prof. Dr. Julio Yarmuch, por su empuje y estímulo, al incentivarme para postular y ser parte del Directorio de la Sociedad de Cirujanos de Chile.
${ }^{1}$ Comité de Educación Sociedad de Cirujanos de Chile

Correspondencia a: camposal33@yahoo.com 